C. D. Andriesse

Kapteyn Astronomical Institute, Roden, The Netherlands

R. Viotti

Laboratorio di Astrofisica Spaziale, Frascati, Italy

ABSTRACT: This high luminosity $\left(5 \times 10^{6} \mathrm{~L} \mathrm{~L}_{\odot}\right)$ star since 1840 is losing mass at the rate of $7.5 \times 10^{-2}$ Mo per year. The large mass loss could be the result of vibrational instabilities produced in the CNO hydrogen burning phase of a very massive $\left(160 \mathrm{M}_{\odot}\right)$ star. The presence of high excitation lines in the ultraviolet spectrum of Eta Car suggests the idea of a hot zone excited by dissipation of the supersonic turbulent flow.

\title{
1. LUMINOSITY
}

The nature and evolutionary stage of Eta Car is still very controversial, because of the many peculiarities of the star. The infrared observations of Neugebauer and Westphal (1968) and others have disclosed that the object is one of the brightest infrared sources of the sky. The integrated energy flux is about $2.7 \times 10^{-8} \mathrm{~W} \mathrm{~m}^{-2}$, the main part of which (99 per cent) being emitted in the infrared. At a distance of $2500 \mathrm{pc}$ this amounts to a luminosity of $5 \times 10^{6} \mathrm{~L}_{\odot}$. The study of the light curve of Eta Car leads to the conclusion that the star has been a very luminous object since at least 150 years. In fact, during its bright phase (18201850) the star had a bolometric magnitude of $-13^{\mathrm{m}}$ (taking into account the interstellar extinction of $\left.E_{B-V}=0.4-0.5\right)$, which is one magnitude brighter than the present bolometric magnitude derived from the infrared observations. Since this difference may be accounted for by the mechanical power required to push off the large mass lost by the star, the conclusion is that the star has no significantly changed its luminosity since its bright stage.

\section{MASS LOSS}

Eta Car is one of the few cosmic objects for which there is clear evidence 47

P. S. Conti and C. W. H. de Loore (eds.), Mass Loss and Evolution of O-Type Stars, 47-50. Copyright 1979 by the IAU. 
for dust condensation in the circumstellar expanding envelope. This problem has been studied in details by Andriesse, Donn and Viotti (1978) who found that dust has been condensing initially at the rate of $10^{-4} \mathrm{M}_{\odot}$ per year, and presently at a somewhat higher rate. For cosmic element abundances in the gas envelope of Eta Car the mass flow from the star should be at least 100 times larger than the condensation rate. Actually it may be one order of magnitude larger, allowing for inefficiencies in the condensation process. On the other hand, the fact that the star is now intrinsically 1 mag fainter than before 1840 implies that, if the nuclear production in the star is unchanged during the last 140 years, 60 per cent of the energy production is presently employed in the expulsion of the gas. The mass flow driven by this power is $0.1 \mathrm{M}_{\odot}$ per year. Andriesse et al. concluded for a mass loss rate of $7.5 \times 10^{-2} \mathrm{M}_{\odot}$ per year, a value which is in agreement with the estimates of the electron density in the ionized region near Eta Car as derived from the spectroscopic observations, and with theoretical considerations discussed below.

\section{MASS AND EVOLUTION}

It is clear that such a large luminosity and mass loss rate rise serious problems in attempting theoretical models for Eta Car. Since the star is located near the center of the young $O B$ association Tmumpler 16, which includes main sequence 03 stars, it should belong to the association. Feinstein et al. (1973) estimated for $\operatorname{Tr} 16$ an age of $3 \times 10^{6}$ years, and this may be the age of Eta Car. Being the most luminous, hence the most massive member of the association, Eta Car should be evolved from the main sequence. According to Chiosi et al. (1978) during the core hydrogen burning, massive stars with large mass loss rates evolving from the main sequence do not join the red giant region. Their evolutionary tracks shrink towards the main sequence, and eventually cross it. The lifetime spent in the core hydrogen buming phase for a $100 \mathrm{M}_{\odot}$ star is $3 \times 10^{6} \mathrm{yr}$, with a mean mass loss rate of $1-2 \times 10^{-5} M_{0}$ per year, more than three orders of magnitude smaller than that of Eta Car. The mass of Eta Car can be estimated from the binding condition that $\mathrm{M}_{*}>\mu \mathrm{L} /(4 \pi \mathrm{cG})$, Andriesse et al. (1978), that is that the star should be stable against radiation pressure. The critical mass is $145 \mathrm{M}_{\odot}$; but in the case of considerable mass loss, the ratio of the radiative to the gravitational acceleration is probably close to unity. We thus estimate a stellar mass some 10 per cent heavier than the above limit, say $160 \mathrm{M}_{\odot}$. A star with such a large mass is not expected to be stable. According to Hoyle et al. (1973) an excess of nuclear energy is produced in these stars during the CNO hydrogen burning phase as a result of temperature variations in stellar pulsations. Thus the large mass loss could be the result of shock waves which take up the excess of nuclear energy. According to Hoyle et al. the 
continuous mass flow would be about $6 \times 10^{-9} \mathrm{M}_{\odot}$ per year. Inserting the complete $\mathrm{L}=2.5 \mathrm{x}$ Lrad of Eta Car we obtain $7.5 \times 10^{-2} \mathrm{M}_{\odot}$ per year.

\section{IUE OBSERVATIONS}

The ultraviolet spectrum of Eta Car may provide useful information about the structure of the expanding envelope and the mass loss. Viotti, Cassatella and Giangrande (1978) have recently observed the high resolution ultraviolet spectrum of Eta Car with the Intermational Ultraviolet Explorer. They have identified many emission lines including the resonance lines of CIV, NV, MgII, AlIII, SiII, SiIV, MnII and FeII which have $P$ Cygni profiles. The broad absorptions extend from about $-230 \mathrm{~km} \mathrm{~s}^{-1}$ to $-650 \mathrm{~km} \mathrm{~s}^{-1}$, confirming the presence of a large velocity gradient in the envelope. The identification of high energy lines, namely HeII, NV, CIV and in the optical region NeIII, in the spectrum of Eta Car may be explained by the presence in the expanding envelope of a hot zone which could be produced by dissipation of the supersonic turbulent flow as suggested by Andriesse et al. (1978).

\section{REFERENCES}

Andriesse, C.D., Donn, B.D. and Viotti, R., 1978, Mon. Not. Roy. Astron. Soc., in press.

Chiosi, C., Nasi, E. and Sreenivasan, S.R., 1978, Astron. Astrophys. 63, 103.

Feinstein, A., Marraco, H.G. and Muzzio, J.C., 1973, Astron. Astrophys. Suppl. 12, 331 .

Hoyle, F., Solomon, P.M. and Woolf, N.J., 1973, Astrophys. J. 185, L89. Neugebauer, G. and Westphal, J.A., 1968, Astrophys. J. 152, L89. Viotti, R., Cassatella, A. and Giangrande, A., 1978, 4th Colloquium on Astrophysics of the Trieste Astronomical Observatory "High Resolution פpectroscopy", Ed. M. Hack, Trieste 3-7 July 1978. 
DISCUSSION FOLLOWING ANDRIESSE AND VIOTTI

Conti: I would like to suggest an alternative explanation for the evolutionary status of $n$ Car. Chiosi has emphasized that the status of an early type star burning $\mathrm{H}$ in the core may be quite different from one burning He in the core. I think $n$ Car might be a star undergoing this "event," changing from one kind of nuclear burning to another. I recognize this is clearly speculative.

Chiosi: I myself am somewhat uncomfortable with the quoted rates. The theoretical rates for mass loss by vibrational instability (Appenzeller) are $10^{-3} \sim 10^{-4}$ solar masses per year. This is two orders of magnitude discrepant. 